\title{
Audio-visual distraction as an adjunct to standby anaesthesia in persons with paraplegia: a case series of five operations
}

\author{
Khurram Ayub $^{1} \cdot$ Ruth A. Corrigan ${ }^{1} \cdot$ Jyoti Misra ${ }^{1} \cdot$ Svetlana Galitzine ${ }^{1}$
}

Received: 11 May 2017 / Revised: 10 November 2017 / Accepted: 11 November 2017

(c) International Spinal Cord Society 2018

\begin{abstract}
Introduction Persons with paraplegia present complex challenges to anaesthetists. Complications experienced by these patients can require major orthoplastic surgery such as excision of infected bone and soft tissue due to pressure sores and soft tissue reconstruction. Anaesthetic strategies deemed both safe and acceptable to this population are essential.

Case presentation We report a case series of five procedures in four patients with complete chronic spinal cord injury (CSCI) who underwent operations in lateral position under standby anaesthesia and audio-visual distraction (AVD) with minimal sedation. Patients' experience was formally assessed as part of the ongoing AVD service evaluation in our institution. All stated that they were not concerned in the operating theatre and felt "comfortable" or "very comfortable" throughout. All patients rated the experience as "better" than their previous experience with general anaesthesia and felt "very satisfied" with their anaesthetic. Importantly, all patients would recommend sedation with AVD to other patients.

Discussion To our knowledge, this is the first report of AVD application as an adjunct to standby anaesthesia during major surgery in persons with paraplegia. Patient feedback was extremely positive, therefore it is likely that by making standby anaesthesia more acceptable to patients the use of AVD could reduce anaesthetic risk in this complex patient group.
\end{abstract}

\section{Introduction}

Patients with chronic spinal cord injury (CSCI) present a challenge to anaesthetists for a variety of reasons, including autonomic dysreflexia, muscle spasms, respiratory weakness, impaired cough, abnormal spinal anatomy and cardiovascular co-morbidities [1, 2]. The extent of these challenges depends on the completeness of transection and level of the injury, as well as pre-existing and new comorbidities. Persons with paraplegia often develop complications requiring surgical intervention, for example, urological procedures and pressure sore debridement [1]. The latter can require major orthoplastic procedures involving excision of infection of bone and soft tissue, insertion of antibiotic carrier and soft tissue closure. Therefore, it is

Electronic supplementary material The online version of this article (https://doi.org/10.1038/s41394-017-0035-6) contains supplementary material, which is available to authorised users.

Khurram Ayub

ayubkhurram@aol.com

1 Nuffield Department of Anaesthetics, Oxford University Hospitals Foundation Trust, Headley Way, Oxford OX3 9DU, UK essential to find safe and acceptable methods to provide surgical anaesthesia to these patients.

Three methods of anaesthesia are recognised in this patient cohort: general, regional and standby anaesthesia. General anaesthesia is effective; however, risk of respiratory dysfunction and autonomic dysreflexia (especially if masked by anaesthetic agents) can make it more challenging in patients with spinal cord injury. Regional anaesthesia can reduce the frequency of autonomic dysreflexia, although the effectiveness of the block can be difficult to assess and there is a small risk of damage to adjacent structures. Finally, standby anaesthesia may be appropriate in patients with a complete sensory deficit undergoing surgery below the level of the lesion; however, its use can be limited by patients' unwillingness to consent due to anxiety and/or concern of awareness of the surgical procedure $[1,2]$.

We report a case series of five procedures in four persons with paraplegia who required orthoplastic surgery for infected pressure sores in lateral position and benefitted from audio-visual distraction (AVD) as part of standby anaesthesia with minimal sedation. The five operations were consecutive and all patients were agreeable to "try" the AVD which has already been well established as an adjunct to regional anaesthesia with positive patients' feedback in our 
Table 1 Summary of patient responses

\begin{tabular}{|c|c|c|c|c|c|}
\hline Case number & 1 & 2 & 3 & 4 & 5 \\
\hline Age & 78 & 64 & 51 & 51 & 53 \\
\hline Sex & Male & Male & Male & Male & Male \\
\hline Paraplegia & T12 complete & & & & C7 complete \\
\hline Type of operation & Leg & Buttocks & Leg & Leg & Leg \\
\hline Length of operation (h) & $1-2$ & $2-4$ & $2-4$ & $1-2$ & $1-2$ \\
\hline Sedation & Yes & Yes & Yes & No & Yes \\
\hline AVD option & $\begin{array}{l}\text { Television and } \\
\text { movies }\end{array}$ & Games and movies & Music & Music & Music \\
\hline Awareness & Not at all & Not at all & Not at all & Not at all & Not at all \\
\hline Comfort & Comfortable & Very comfortable & Very comfortable & Very comfortable & $\begin{array}{l}\text { Very } \\
\text { comfortable }\end{array}$ \\
\hline Effect on anxiety & I was not anxious & I was not anxious & I was not anxious & I was not anxious & $\begin{array}{l}\text { I was not } \\
\text { anxious }\end{array}$ \\
\hline Compared to GA & Better & Much better & Better & Better & Better \\
\hline Satisfaction & Very satisfied & Very satisfied & Very satisfied & Very satisfied & Very satisfied \\
\hline $\begin{array}{l}\text { Recommendation to } \\
\text { others }\end{array}$ & Yes & Yes & Yes & Yes & Yes \\
\hline \multirow[t]{4}{*}{ Comments } & & $\begin{array}{l}\text { Felt hammering of bone, } \\
\text { not alarming }\end{array}$ & $\begin{array}{l}\text { Anaesthetist very lovely and } \\
\text { nice. Pleasant to talk to }\end{array}$ & $\begin{array}{l}\text { FM album by } \\
\text { SKINTS }\end{array}$ & Fantastic \\
\hline & & Awake as soon as finished & & & \\
\hline & & No prolonged recovery & & & \\
\hline & & $\begin{array}{l}\text { Feels that more people } \\
\text { should use this technique }\end{array}$ & & & \\
\hline
\end{tabular}

institution. To our knowledge, these are the first reported cases of this kind. Our experience shows that application of AVD is a very useful strategy to make standby anaesthesia more acceptable to this complex patient population.

\section{Case presentation}

Case \#1 involved a 78-year-old gentleman with a T10 complete transection after a traumatic spinal fracture 4 years previously. He developed a pressure sore on the posterior aspect of his left thigh with MRI suggesting femoral osteomyelitis. There were no co-morbidities. Following premedication with $20 \mathrm{mg}$ oral temazepam he underwent surgical excision of infected bone and soft tissues and insertion of an antibiotic carrier into the bone defect by orthopaedic surgeons and local hamstring advancement for soft tissue closure by plastic surgeons. Standby anaesthesia for a $2 \mathrm{~h}$ procedure involved conscious sedation with $2 \mathrm{mg}$ of midazolam in total and AVD, the latter in a form of watching a movie on the internet-connected tablet. Noisereducing headphones were part of AVD kit.

Case \#2 involved a 59-year-old gentleman with a traumatic T7/8 complete spinal transection 35 years previously. He was bacteraemic, with a discharging pressure sore over his left ischium. Comorbidities included controlled atrial fibrillation (on digoxin and warfarin) and diabetes (on metformin). He underwent a $2 \mathrm{~h}$ and 30 min excision of the ischial osteomyelitis and local hamstring advancement under minimal sedation (midazolam $3 \mathrm{mg}$ and propofol TCI $300 \mathrm{mg}$ total) and AVD (games and movies). Apart from requiring five $0.5 \mathrm{mg}$ aliquots of metaraminol, anaesthesia was uneventful.

Case \#3 and Case \#4 involved two procedures in a 51year-old gentleman with paraplegia secondary to spina bifida. He presented with infected ischial pressure sore, comorbidities included controlled asthma and hypertension. His $2 \mathrm{~h}$ and $40 \mathrm{~min}$ procedure-excision of the pressure ulcer, resection of the underlying ischial tuberosity and ramus and a local muscle flap-was performed under minimal sedation (midazolam $1.5 \mathrm{mg}$ and propofol TCI $0.5-1 \mathrm{mcg} / \mathrm{ml}$ ) and AVD (music). A week later the patient required $1 \mathrm{~h}$ and $5 \mathrm{~min}$ procedure-evacuation of postoperative haematoma. He opted to use his own generic portable telephone for AVD and declined sedation throughout the procedure.

Case \#5 involved a 53-year-old gentleman with paraplegia secondary to traumatic $\mathrm{C} 7$ complete spinal transection. He presented with grade 4 pressure sore over his left hip, comorbidities included hepatitis $\mathrm{C}$ and sarcoidosis. His 
$2 \mathrm{~h}$ and $5 \mathrm{~min}$ procedure-excision of the pressure ulcer, resection of the underlying bone and a local muscle flapwas performed under minimal sedation (midazolam $0.5 \mathrm{mg}$ and propofol TCI $0.4-0.6 \mathrm{mcg} / \mathrm{ml}$ ) and AVD (music). He opted to use his own generic portable tablet for distraction during the procedure.

Perioperatively, all patients consented to standardised questioning (Appendix 1) and informed consent to publication was obtained about their experience with AVD with or without sedation. All stated that they were not concerned in the operating theatre and that they felt "comfortable" or "very comfortable" throughout. Of note, one patient commented that he was aware of the surgeons "hammering the bone" but was not alarmed by this. All patients rated the experience as "better" than their previous experience with general anaesthesia and felt "very satisfied" with their anaesthetic. Importantly, all patients would recommend sedation with AVD to other patients. One patient cited "no prolonged recovery" as the main advantage of this technique; another highlighted his "pleasant" chat with the anaesthetist throughout the procedure; and another described the experience as "fantastic" (Table 1).

All patients received standard non-invasive monitoring, intravenous crystalloids and supplemental oxygen via Hudson mask or nasal prongs. All procedures were carried out in a "sloppy" lateral position, a half-way position between conventional lateral decubitus position for hip replacement and prone position, adopted by the surgical and anaesthetic team as a compromise allowing adequate surgical access and preservation of unassisted spontaneous respiration and comfort of an awake patient. There were no anaesthesia-related problems. All patients were managed postoperatively in a specialised Bone Infection Ward and discharged from the hospital.

\section{Discussion}

This case series highlights the benefits of AVD when complimenting suitable anaesthesia with conscious sedation in patients with complete CSCI requiring surgical procedures below the level of injury. Though AVD is a known useful adjunct to regional anaesthesia, this is the first report of it being used in persons with paraplegia.

This technique was used in reported patients for four complex orthoplastic operations and, in one of the patients, for a shorter procedure, an evacuation of post-operative haematoma. Operations required access to the posterior-lateral parts of the pelvis or thigh. As reported above, the "sloppy" lateral position was used by the surgeons on anaesthetists' request as a compromise between patient comfort, safety and surgical access. This position avoided the use of prone position that could have required general anaesthesia with intubation. Remarkably, the lateral position did not preclude watching films or playing games on a touch screen tablet.

Our series presents five operations in four consecutive patients, with all patients being agreeable to the proposed technique. In case of a patient not agreeing to AVD and sedation, the alternative would have been a general anaesthetic with intubation and ventilation which was considered the safest "Plan B" option for this type of operations.

Following institutional approval and patients' consent, the patients were questioned about their anaesthetic experience using a standardised questionnaire. All four rated their experience highly and preferred this method of anaesthesia to their previous general anaesthetics, citing the quicker recovery in the post-operative period as the main advantage. None found the experience uncomfortable. Indeed, when needing to return to theatre for the evacuation of a haematoma, one of the patients happily underwent the repeat procedure without any sedation and with used his own smart phone with downloaded music of his choice.

Compared to general anaesthesia, conscious sedation offers many potential advantages including faster recovery time and return to eating and drinking, and reduced postoperative nausea and vomiting. Most importantly, patients are not ventilated, and therefore there is lesser risk of cardio-pulmonary complications. However, many patients find the concept of being "awake" during an operation frightening [3]. The use of distraction in the operating theatre is becoming increasingly popular, with evidence that video distraction reduces anxiety of those undergoing operations [4, 5]. In addition, audio and AVD have also been shown to reduce the need for patient controlled sedative and analgesia during invasive procedures $[3,6,7]$. This is a particular advantage in persons with paraplegia who may have co-existent autonomic dysfunction or respiratory compromise perioperatively.

More recently, the use of AVD in combination with regional anaesthesia has become increasingly popular, with some patients requesting AVD and regional anaesthesia in preference to general anaesthesia in some centres [8-11]. While patients with complete CSCI do not require intraoperative regional anaesthesia, their fears and anxiety about surgery and environment can be as bad as those of patients having surgery under local or regional anaesthesia, and the risk of being oversedated and respiratory compromised is, perhaps, greater than in general population.

The described technique has become possible due to multimedia equipment for AVD which has recently become available in our orthopaedic centre and our growing positive experience of AVD and regional anaesthesia case series in patients undergoing variety of minor and major orthopaedic and plastic limb procedures, including prolonged orthoplastic procedures [12-14]. Our AVD kit consists of a Wi- 
Fi connected tablet device with additional preloaded selection of music and movies, and noise-reducing headphones. The formal service evaluation showed very positive patients reported outcomes [15, 16]. We feel that our positive experience with AVD in general population could be translated into the specific population of CSCI patients. It would be interesting, in future, to use more sophisticated devices like "virtual reality" to verify the results.

In summary, this five operations case study extends published literature on use of AVD to include major surgery in persons with paraplegia under standby anaesthesia. It is likely that the use of AVD will make standby anaesthesia more attractive to persons with paraplegia, thus reducing the anaesthetic risk in this already complex patient group. Further work is needed to quantify the predicted reduction in immediate post-operative recovery and cardio-respiratory complications compared to use of general anaesthesia in this patient cohort.

\section{Compliance with ethical standards}

Conflict of interest The authors declare that they have no conflict of interest.

\section{References}

1. Hambly PR, Martin B. Anaesthesia for chronic spinal cord lesions. Anaesthesia 1998;53:273-89.

2. Petsas A, Drake J. Perioperative management for patients with a chronic spinal cord injury. Br J Anaesth Educ 2015;15:123-30.

3. Mitchell M. Conscious surgery: influence of the environment on patient anxiety. J Adv Nurs 2008;64:261-71.

4. Man AKW, Yap JCM, Kwan SY, Suen KL, Yip HS, Chen PP. The effect of intra-operative video on patient anxiety. Anaesthesia 2003;58:64-8.
5. Wismeijer AA, Vingerhoets AJ. The use of virtual reality and audiovisual eyeglass systems as adjunct analgesic techniques: a review of the literature. Ann Behav Med 2005;30:268-78.

6. Koch ME, Kain ZN, Ayoub C, Rosenbaum SH. The sedative and analgesic sparing effect of music. Anesthesiology 1998;89:300-6.

7. Lee DWH, Chan ACW, Wong SKH, Fung TMK, Li CAN, Mui $\mathrm{LM}$, et al. Can visual distraction decrease the dose of patient controlled sedation required during colonoscopy? A prospective randomized controlled trial. Endoscopy 2004;36:197-201.

8. O'Connor K, Pace N. Audio-visual distraction during spinal anaesthesia for orthopaedic surgery. $\mathrm{J}$ Perioper Pract 2010;20:428-9.

9. Schecter WP, Farmer D, Horn JK, Pietrocola DM, Wallace A. Special considerations in perioperative pain management: audiovisual distraction, geriatrics, pediatrics, and pregnancy. J Am Coll Surg 2005;201:612-8

10. Schneider SM, Prince-Paul M, Allen MJ, Silverman P, Talaba D. Virtual reality as a distraction intervention for women receiving chemotherapy. Oncol Nurs Forum 2004;31:81-8.

11. Wu F, Shahid M, Waldram M, Audiovisual distraction as an adjunct to anxiety relief in hand surgery with regional anaesthesia. J Hand Surg Eur 2013;38:1000-1.

12. Athanassoglou V, Galitzine S, Choi D, Matthews J, Patients' reported experience with novel audiovisual distraction technique for prolonged upper limb surgery under regional anaesthesia. Reg Anaesth Pain Med 2014;39:E227.

13. Athanassoglou V, Wallis A, Galitzine S. Audio-visual distraction as a useful adjunct to epidural anaesthesia and sedation for prolonged lower limb microvascular orthoplastic surgery. J Clin Anesth 2015;27:606-11.

14. Misra J, Galitzine S, Athanassoglou V, Pepper W, Ramsden A, McNally M. Audiovisual distraction as an adjunct to epidural anaesthesia in "awake" patients undergoing complex surgery for osteomyelitis. Bone Jt J 2016;98-B:60.

15. Bajaj A, Hsu D. A prospective study monitoring the effects of audio and visual distractions on patients undergoing surgical procedures with regional anaesthesia. Anesthesiology 2004;101: A516.

16. Pepper W, Athanassoglou V, Matthews J, Galitzine S. A survey of 50 patients undergoing awake surgery with audiovisual distraction, assessing the patient experience and comparing it to previous general anaesthesia. Reg Anaesth Pain Med 2016;41:E156. 